\title{
GROWTH OF RANK 1 VALUATION SEMIGROUPS
}

\author{
STEVEN DALE CUTKOSKY, KIA DALILI AND OLGA KASHCHEYEVA
}

Let $\left(R, m_{R}\right)$ be a local domain, with quotient field $K$. Suppose that $\nu$ is a valuation of $K$ with valuation ring $\left(V, m_{V}\right)$, and that $\nu$ dominates $R$; that is, $R \subset V$ and $m_{V} \cap R=m_{R}$. The possible value groups $\Gamma$ of $\nu$ have been extensively studied and classified, including in the papers MacLane [8], MacLane and Schilling [9], Zariski and Samuel [12], and Kuhlmann [7. $\Gamma$ can be any ordered abelian group of finite rational rank (Theorem 1.1 [7]). The semigroup

$$
S^{R}(\nu)=\left\{\nu(f) \mid f \in m_{R} \backslash\{0\}\right\}
$$

is however not well understood, although it is known to encode important information about the topology and resolution of singularities of $\operatorname{Spec}(R)$ and the ideal theory of $R$.

In Zariski and Samuel's classic book on Commutative Algebra [12], two general facts about the semigroup $S^{R}(\nu)$ are proven (in Appendix 3 to Volume II).

1. $S^{R}(\nu)$ is a well ordered subset of the positive part of the value group $\Gamma$ of $\nu$ of ordinal type at most $\omega^{h}$, where $\omega$ is the ordinal type of the well ordered set $\mathbb{N}$, and $h$ is the rank of $\nu$.

2. The rational rank of $\nu$ plus the transcendence degree of $V / m_{V}$ over $R / m_{R}$ is less than or equal to the dimension of $R$.

The second condition is the Abhyankar inequality [1].

The only semigroups which are realized by a valuation on a one dimensional regular local ring are isomorphic to the natural numbers. The semigroups which are realized by a valuation on a regular local ring of dimension 2 with algebraically closed residue field are much more complicated, but are completely classified by Spivakovsky in [10]. A different proof is given by Favre and Jonsson in [5], and the theorem is formulated in the context of semigroups by Cutkosky and Teissier [3].

In [3], Teissier and the first author give some examples showing that some surprising semigroups of rank $>1$ can occur as semigroups of valuations on noetherian domains, and raise the general questions of finding new constraints on value semigroups and classifying semigroups which occur as value semigroups.

In this paper, we consider semigroups of rank 1 valuations. We show in Theorem 2.1 that the Hilbert polynomial of $R$ gives a bound on the growth of the valuation semigroup $S^{R}(\nu)$. This allows us to give (in Corollary 2.4) a very simple example of a well ordered subsemigroup of $\mathbb{Q}_{+}$of ordinal type $\omega$, which is not a value semigroup of a local domain. This shows that the above conditions 1 and 2 do not characterize value semigroups on local domains.

The simple bound of Theorem 2.1 of this paper is extended in the article [4] of Teissier and the first author to give a very general bound on the growth of a value semigroup of arbitrary rank, from which a rough description of the (extremely bizzare) shape of a higher rank valuation semigroup is derived.

The first author was partially supported by NSF . 
Prior to this paper and [4, no other general constraints were known on the value semigroups $S^{R}(\nu)$. In fact, it was even unknown if the above conditions 1 and 2 characterize value semigroups.

With our restriction that $\nu$ has rank 1 , we can assume that $S^{R}(\nu)$ is embedded in $\mathbb{R}_{+}$. We can further assume that $s_{0}=1$ is the smallest element of $S^{R}(\nu)$. For $n \in \mathbb{N}$, let

$$
\varphi(n)=\left|S^{R}(\nu) \cap(0, n)\right| .
$$

Corollary 2.2 of Theorem 2.1 shows that for $n \gg 0$,

$$
\varphi(n)<P_{R}(n)
$$

where $P_{R}(n)$ is the Hilbert polynomial of $R$. This bound is the best possible for a one dimensional local domain, as we show after Corollary 2.4. However, this bound is far from being sharp for $R$ of dimension larger than one. Let

$$
\mathcal{P}_{n}=\{f \in R \mid \nu(f) \geq n\}
$$

an ideal in $R$ which contains $m_{R}^{n}$. Suppose that $R$ contains a field $k$ isomorphic to $R / m_{R}$, and $R / m_{R} \cong V / m_{V}$. Then for $n \gg 0$,

$$
\varphi(n)=P_{R}(n)-\ell\left(\mathcal{P}_{n} / m_{R}^{n}\right),
$$

where $\ell\left(\mathcal{P}_{n} / m_{R}^{n}\right)$ is the length of $\mathcal{P}_{n} / m^{n}$. We approximate $\ell\left(\mathcal{P}_{n} / m_{R}^{n}\right)$ to show in Corollary 3.4 that

$$
\lim \sup \frac{\varphi(n)}{n^{d}}<\frac{e(R)}{d !}=\lim _{n \rightarrow \infty} \frac{P_{R}(n)}{n^{d}}
$$

whenever $R$ has dimension $d \geq 2$, where $e(R)$ is the multiplicity of $R$. When the dimension $d$ of $R$ is greater than 1 , this is significantly smaller than the upper bound given by the Hilbert polynomial $P_{R}(n)$ of $R$.

In Section 4, we consider the rates of growth which are possible for the function $\varphi(n)$. We show that quite exotic behavior can occur, giving examples (Examples 4.4, 4.5] and 4.6) of valuations $\nu$ dominating a regular local ring $R$ of dimension two which have growth rates $n^{\alpha}$ for any $\alpha \in \mathbb{Q}$ with $1 \leq \alpha \leq 2$. We also give an example of $n \log n$ growth in Example 4.7.

In the final section, we consider the general question of characterizing rank 1 value semigroups, and ask if the necessary condition on a well ordered subsemigroup $S$ of $\mathbb{R}_{+}$ that the growth of $|S \cap(0, n)|$ is bounded above by a polynomial in $n$ is sufficient for $S$ to be a valuation semigroup.

\section{Notation}

The following conventions will hold throughout this paper.

If $G$ is a totally ordered abelian group, then $G_{+}$will denote the positive elements of $G$. $G_{\geq 0}$ will denote the non negative elements. If $a, b \in G$, we set

$$
(a, b)=\{x \in G \mid a<x<b\} .
$$

$\left(R, m_{R}\right)$ will denote a (Noetherian) local ring with maximal ideal $m_{R}$, and $\ell(N)$ will denote the length of an $R$ module $N$. Let

$$
P_{R}(n)=\frac{e(R)}{d !} n^{d}+\text { lower order terms }
$$

be the Hilbert Samuel polynomial of $R$, where $d$ is the dimension of $R$ and $e(R)$ is the multiplicity of $R$. We have that $\ell\left(R / m_{R}^{n}\right)=P_{R}(n)$ for $n \gg 0$. 
Suppose that $R$ is a local domain with quotient field $K$, and $\nu$ is a valuation of $K$ with valuation ring $\left(V, m_{V}\right)$. We will say that $\nu$ dominates $R$ if $R \subset V$ and $m_{V} \cap R=m_{R}$. We define the value semigroup of $\nu$ on $R$ to be

$$
S^{R}(\nu)=\nu\left(m_{R}-\{0\}\right) .
$$

Let $\Gamma$ be the valuation group of $\nu$. For $\lambda \in \Gamma$, we define ideals in $R$

$$
\mathcal{P}_{\lambda}=\{f \in R \mid \nu(f) \geq \lambda\} .
$$

\section{BOUNDS FOR GROWTH OF SEMigroups OF RANK 1 VALUATIONS}

The bounds in this section are valid for valuations of arbitrary rank, but since they give information about the smallest segment of the value group, they are essentially statements about rank 1 valuations. We use here, and in the remainder of this paper the notation introduced above in Section 1 .

Theorem 2.1. Suppose that $R$ is a local domain which is dominated by a valuation $\nu$, and suppose that $s_{0}$ is the smallest element of $S^{R}(\nu)$. Then

$$
\left|S^{R}(\nu) \cap\left(0, n s_{0}\right)\right|<\ell\left(R / m_{R}^{n}\right)
$$

for all $n \in \mathbb{N}$.

Proof. Suppose that $n \in \mathbb{N}$. Since $S^{R}(\nu)$ is well ordered, $\left(0, n s_{0}\right) \cap S^{R}(\nu)$ is a finite set

$$
\lambda_{1}<\cdots<\lambda_{r}
$$

for some $r \in \mathbb{N}$. Set $\lambda_{r+1}=n s_{0}$. We have a sequence of inclusions of ideals (as defined in Section 1)

$$
m_{R}^{n} \subset \mathcal{P}_{n s_{0}}=\mathcal{P}_{\lambda_{r+1}} \subset \mathcal{P}_{\lambda_{r}} \subset \cdots \subset \mathcal{P}_{\lambda_{1}}=m_{R}
$$

Thus

$$
\sum_{i=1}^{r} \ell\left(\mathcal{P}_{\lambda_{i}} / \mathcal{P}_{\lambda_{i+1}}\right) \leq \ell\left(m_{R} / m_{R}^{n}\right) .
$$

Since $\ell\left(\mathcal{P}_{\lambda_{i}} / \mathcal{P}_{\lambda_{i+1}}\right)>0$ for all $i$, we have the desired inequality.

Recall that $P_{R}(n)$ is the Hilbert polynomial of a local ring $R$.

Corollary 2.2. Suppose that $R$ is a local domain of dimension $d$ which is dominated by a valuation $\nu$, and $s_{0}$ is the smallest element of $S^{R}(\nu)$. Then

1. For all positive integers $n \gg 0,\left|S^{R}(\nu) \cap\left(0, n s_{0}\right)\right|<P_{R}(n)$.

2. There exists $c>0$ such that $\left|S^{R}(\nu) \cap\left(0, n s_{0}\right)\right|<c n^{d}$ for all $n \in \mathbb{N}$.

Corollary 2.3. Suppose that $R$ is a regular local ring of dimension $d$ which is dominated by a valuation $\nu$, and $s_{0}$ is the smallest element of $S^{R}(\nu)$. Then

$$
\left|S^{R}(\nu) \cap\left(0, n s_{0}\right)\right|<\left(\begin{array}{c}
d-1+n \\
d
\end{array}\right)
$$

for all $n \in \mathbb{N}$.

Corollary 2.4. There exists a well ordered subsemigroup $U$ of $\mathbb{Q}_{+}$such that $U$ has ordinal type $\omega$ and $U \neq S^{R}(\nu)$ for any valuation $\nu$ dominating a local domain $R$. 
Proof. Take any subset $T$ of $\mathbb{Q}_{+}$such that 1 is the smallest element of $T$ and $n^{n} \leq$ $|T \cap(0, n)|<\infty$ for all $n \in \mathbb{N}$. For all positive integers $r$, let

$$
r T=\left\{a_{1}+\cdots+a_{r} \mid a_{1}, \ldots, a_{r} \in T\right\} .
$$

Let $U=\omega T=\cup_{r=1}^{\infty} r T$ be the semigroup generated by $T$. By our constraints, $|U \cap(0, r)|<$ $\infty$ for all $r \in \mathbb{N}$. Thus $U$ is well ordered and has ordinal type $\omega$. By 2 of Corollary 2.2, $U$ cannot be the semigroup of a valuation dominating a local domain.

We will now consider more closely the bound

$$
\left|S^{R}(\nu) \cap\left(0, n s_{0}\right)\right|<P_{R}(n)
$$

for $n \gg 0$ of 1 of Corollary 2.2.

In the case when $R$ is a regular local ring of dimension 1, we have that

$$
\left|S^{R}(\nu) \cap\left(0, n s_{0}\right)\right|=n-1=P_{R}(n)
$$

for all $n \in \mathbb{N}$, so that the bound (31) is sharp.

When $R$ is an arbitrary local domain of dimension 1, the bound (3) can be far from sharp, as is shown by the following example. Let $R$ be the localization of $k[x, y] / y^{2}-x^{2}-x^{3}$ at the maximal ideal $(x, y)$. Define a valuation $\nu$ which dominates $R$ by embedding $R$ into the power series ring $k[[t]]$ by the $k$-algebra homomorphism which maps $x$ to $t$ and $y$ to $t \sqrt{1+t}$. Let $\nu$ be the restriction of the $t$-adic valuation to $R$. Then $S^{R}(\nu)=\mathbb{N}$ and $s_{0}=1$, and $\left|S^{R}(\nu) \cap\left(0, n s_{0}\right)\right|=n-1$ for all positive $n$. But $R$ has multiplicity 2 , and $P_{R}(n)-1=2(n-1)$ for all positive $n$.

However, (3) can be sharp for a one dimensional $R$ which is not regular, as is illustrated by the following example. Let $R$ be the localization of $k[x, y] / y^{2}-x^{3}$ at the maximal ideal $(x, y)$. Embed $R$ into the valuation ring $V=k[t]_{(t)}$ by the $k$-algebra homomorphism which maps $x$ to $t^{2}$ and $y$ to $t^{3}$. Let $\nu$ be the restriction of $\frac{1}{2}$ times the $t$-adic valuation on $V$ to $R$. Then $\nu(x)=1, \nu(y)=\frac{3}{2}$ and $S^{R}(\nu)=\left\{1, \frac{3}{2}, 2, \frac{5}{2}, 3, \frac{7}{2}, \ldots\right\}$. Thus $s_{0}=1$ and

$$
\left|S^{R}(\nu) \cap\left(0, n s_{0}\right)\right|=2(n-1)=P_{R}(n)-1
$$

for all positive $n$.

Evidently, in the case of one dimensional domains, (3) is the best bound which is always valid.

In rings of dimension 2 and higher, (3) is always far from sharp, as we show in the next section.

\section{A SHARPER BOUND}

In this section, we assume that $\left(R, m_{R}\right)$ is a local domain of dimension $d$ and multiplicity $e=e(R)$. Suppose $\nu$ is a rank 1 valuation on the quotient field of $R$ with valuation ring $V$ such that $\nu$ dominates $R$, and $R$ contains an infinite field $k$ such that $k \cong R / m_{R}$ with $R / m_{R} \cong V / m_{V}$. Without loss of generality, we may assume that the smallest value of an element of $m_{R}$ is $s_{0}=1$.

Let

$$
\varphi(n)=\left|S^{R}(\nu) \cap(0, n)\right|
$$

for $n \in \mathbb{N}$. We will measure the deviation of $\varphi(n)$ from the upper bound (3) given by the Hilbert-Samuel polynomial $P_{R}(n)$ of $R$.

We begin with another look at the proof of Theorem 2.1 with these assumptions on $R$. Since $k=V / m_{V}$, we have

$$
\ell\left(\mathcal{P}_{\lambda_{i}} / \mathcal{P}_{\lambda_{i+1}}\right)=1
$$


for all $i$ in the sequence (11). For $n \in \mathbb{N}$, let

$$
\psi(n)=\ell\left(\mathcal{P}_{n} / m^{n}\right) .
$$

$\psi(n)$ measures the difference of $\psi(n)$ from the Hilbert-Samuel function as

$$
\varphi(n)=\ell\left(R / m^{n}\right)-\psi(n)
$$

for all $n \in \mathbb{N}$ (by (2) ), and thus

$$
\varphi(n)=P_{R}(n)-\psi(n)
$$

for $n \gg 0$.

Let $A=\operatorname{gr}_{m}(R)$ be the associated graded ring of $R$ and for nonzero $x \in m^{i} \backslash m^{i+1}$, let $\bar{x}$ denote the image of $x+m^{i+1}$ in $A$. We will call $\bar{x}$ the inital form of $x$ and $i$ the initial degree of $x$.

Lemma 3.1. There exist $x_{1}, \ldots, x_{d} \in m \backslash m^{2}$ such that $\bar{x}_{i} \in A$ form an algebraically independent set over $k$, and $\nu\left(x_{i}\right) \neq \nu\left(x_{j}\right)$ for $i \neq j$.

Proof. Since $k$ is infinite, $A$ has a Noether Normalization in degree one. Let $y_{1}, \ldots, y_{d}$ be elements of $m \backslash m^{2}$ such that $k\left[\bar{y}_{1}, \ldots, \bar{y}_{d}\right]$ is a Noether Normalization of $A$.

Since $\nu(a)=\nu(b)$ implies the existence of $\lambda \in k$ such that $\nu(a+\lambda b)>\nu(a)$, we can find $\lambda_{i j} \in k$ such that $x_{i}=\sum \lambda_{i j} y_{j}$ satisfy the desired properties.

Lemma 3.2. Let $x_{i}$ 's be as in the previous lemma, and let $K$ be the fraction field of $k\left[x_{1}, \ldots, x_{d}\right]$. Then there are elements $m_{1}, \ldots, m_{e} \in R$ (where the multiplicity of $R$ is e) such that $\bar{m}_{1}, \ldots, \bar{m}_{e} \in A$ are linearly independent over $K$.

Proof. For $n \gg 0, \ell\left(m^{n} / m^{n+1}\right)$ is a polynomial $Q(n)$ of degree $d-1$. We will compute the leading coefficient of $Q(n)$ in two ways.

Let $B=k\left[\bar{x}_{1}, \ldots, \bar{x}_{n}\right]$. First, observe that $A$ is a finitely generated graded module over the standard graded ring $B$. Since $A$ has dimension $d$, and $B$ has multiplicty one, we can compute from tensoring a graded composition series of $A$ as a $B$ module with $K$ (or from the graded version of the additivity formula given for instance in Corollary 4.7.8 [2]) that the multiplicity of $A$ as a $B$ module is $\operatorname{dim}_{K}\left(A \otimes_{k\left[\overline{x_{1}}, \ldots, \overline{x_{d}}\right]} K\right)$.

For $n \gg 0, Q(n)=P_{R}(n+1)-P_{R}(n)$. Thus

$$
Q(n)=\frac{e}{(d-1) !} n^{d-1}+\text { lower order terms, }
$$

and we conclude that

$$
\operatorname{dim}_{K}\left(A \otimes_{k\left[\overline{x_{1}}, \ldots, \overline{x_{d}}\right]} K\right)=e .
$$

Choose a basis for the vector space $A \otimes_{k\left[\overline{x_{1}}, \ldots, \overline{x_{d}}\right]} K$ of elements of the form $\bar{m}_{i} \otimes 1$. Such $m_{i}$ 's have the desired property.

Proposition 3.3. Suppose the $x_{i}$ 's and $m_{i}$ 's are as in the previous lemmas. Then the infimum limit

$$
\lim \inf \frac{\ell\left(\mathcal{P}_{n} / m^{n}\right)}{n^{d}} \geq \frac{e}{d !}\left(1-\frac{1}{\nu\left(x_{1}\right) \ldots \nu\left(x_{d}\right)}\right) .
$$

Proof. Let $\alpha_{i}$ be the initial degree of $m_{i}$. Let

$$
S=\left\{m_{i} x_{1}^{n_{1}} \cdots x_{d}^{n d} \mid \alpha_{i}+n_{1}+\cdots+n_{d}<n\right\} .
$$


We will first show that the classes of the elements of $S$ in $\mathcal{P}_{n} / m^{n}$ are linearly independent over $k$. Suppose otherwise. Then there is a nontrivial sum

$$
\sum \lambda_{i, n_{1}, \ldots, n_{d}} m_{i} x_{1}^{n_{1}} \cdots x_{d}^{n_{d}} \in m^{n}
$$

where $\alpha_{i}+n_{1}+\cdots+n_{d}<n$ and $0 \neq \lambda_{i, n_{1}, \ldots, n_{d}} \in k$ for all terms in the sum. Let $\tau$ be the smallest value of $\alpha_{i}+n_{1}+\cdots+n_{d}$ for a term appearing in (4).

Since $\tau<n$, we have that

$$
\sum_{\alpha_{i}+n_{1}+\cdots+n_{d}=\tau} \lambda_{i, n_{1}, \ldots, n_{d}} m_{i} x_{1}^{n_{1}} \cdots x_{d}^{n_{d}} \in m^{\tau+1},
$$

and thus

$$
\sum_{\alpha_{i}+n_{1}+\cdots+n_{d}=\tau} \lambda_{i, n_{1}, \ldots, n_{d}} \bar{m}_{i} \bar{x}_{1}^{n_{1}} \cdots \bar{x}_{d}^{n_{d}}=0
$$

in $m^{\tau} / m^{\tau+1} \subset A$. But by Lemma 3.2 , the elements $\bar{m}_{i} \bar{x}_{1}^{n_{1}} \cdots \bar{x}_{d}^{n_{d}}$ are linearly independent over $k$ in $A$, which is a contradiction.

Our next goal is to determine which of the elements of $S$ are in $\mathcal{P}_{n} \backslash m^{n}$. Note that since the classes of these elements in $\mathcal{P}_{n} / m^{n}$ are linearly independent over $k$, their number gives a lower bound on $\ell\left(\mathcal{P}_{n} / m^{n}\right)$.

For a fixed $i$, the condition that an element $m_{i} x_{1}^{n_{1}} \ldots x_{d}^{n_{d}}$ is in $\mathcal{P}_{n} \backslash m^{n}$ can be written as the following system of linear inequalities in terms of $n_{i}$ 's:

$$
\begin{aligned}
\nu\left(m_{i}\right)+\nu\left(x_{1}\right) n_{1}+\cdots+\nu\left(x_{d}\right) n_{d} & \geq n \\
\alpha_{i}+n_{1}+\cdots+n_{d} & <n .
\end{aligned}
$$

Now since $\alpha_{i} \leq \nu\left(m_{i}\right)$ every solution to the following two inequalities is also a solution to the above system.

$$
\begin{array}{ll}
\nu\left(x_{1}\right) n_{1}+\cdots+\nu\left(x_{d}\right) n_{d} & \geq n-\alpha_{i} \\
n_{1}+\cdots+n_{d} & <n-\alpha_{i} .
\end{array}
$$

We will now make an asymptotic approximation of the number of integral solutions to the system (5). To a polytope $P \subset \mathbb{R}^{d}$ and $n \in \mathbb{Z}_{+}$, we associate the Ehrhart function

$$
E(p, n)=\left|\left\{z \in \mathbb{Z}^{d} \mid \frac{z}{n} \in P\right\}\right| .
$$

By approximating $P$ with $d$-cubes of small volume, we compute the volume of $P$ as

$$
\operatorname{vol}(P)=\lim _{n \rightarrow \infty} \frac{E(P, n)}{n^{d}}=\lim _{n \rightarrow \infty} \frac{\left|\left\{z \in \mathbb{Z}^{d} \mid z \in n P\right\}\right|}{n^{d}} .
$$

The volume of the $d$-simplex $\Delta$ with vertices at the origin and at distance $c_{1}, \ldots, c_{d}$ along the coordinate axes is

$$
\operatorname{vol}(\Delta)=\frac{1}{d !} c_{1} \cdots c_{d}
$$

Let $\sigma(n)$ be the set of integral solutions to the system (5). We have that

$$
\begin{aligned}
\lim \inf \frac{\ell\left(\mathcal{P}_{n} / m^{n}\right)}{n^{d}} \geq & \lim _{n \rightarrow \infty} \frac{\sigma(n)}{n^{d}} \\
= & \lim _{n \rightarrow \infty} \frac{\left|\left\{\left(n_{1}, \ldots, n_{d}\right) \in \mathbb{N}^{d} \mid n_{1}+\cdots+n_{d}<n\right\}\right|}{n^{d}} \\
& -\lim _{n \rightarrow \infty} \frac{\left\{\left(n_{1}, \ldots, n_{d}\right) \in \mathbb{N}^{d} \mid \nu\left(x_{1}\right) n_{1}+\cdots+\nu\left(x_{d}\right) n_{d}<n\right\} \mid}{n^{d}} \\
= & \frac{1}{d !}\left(1-\frac{1}{\nu\left(x_{1}\right) \cdots \nu\left(x_{d}\right)}\right) .
\end{aligned}
$$


Corollary 3.4. Let assumptions be as introduced in the beginning of this section. If the first $d$ elements in $S^{R}(\nu)$ are $1=s_{1}, s_{2}, \ldots, s_{d}$, then the supremum limit

$$
\lim \sup \frac{\varphi(n)}{n^{d}}<\frac{e}{d !} \frac{1}{s_{1} \ldots s_{d}}
$$

and thus, if $d>1$,

$$
\lim \sup \frac{\varphi(n)}{n^{d}}<\frac{e}{d !}=\lim _{n \rightarrow \infty} \frac{P_{R}(n)}{n^{d}} .
$$

Proof. From the proposition it follows that there are elements $x_{1}, \ldots x_{d} \in m$ such that $\nu\left(x_{i}\right) \neq \nu\left(x_{j}\right)$ and the number of elements in $S^{R}(\nu) \cap(0, n)$ is asymptotically smaller than

$$
\frac{e}{d !} \frac{1}{\nu\left(x_{1}\right) \ldots \nu\left(x_{d}\right)} n^{d}
$$

Since $x_{i}$ 's have distinct values, we have $s_{1} \ldots s_{d} \leq \nu\left(x_{1}\right) \ldots \nu\left(x_{d}\right)$.

\section{The RATE OF GRowth of VAlue SEMigroups}

In this section, we study the rate of growth of $\varphi(n)=\left|S^{R}(\nu) \cap(0, n)\right|$ when $R$ is a regular local ring of dimension two. We show that a wide range of interesting growth occurs within the possible ranges of $n$ and $n^{2}$.

We say that $\varphi(n)$ has the growth rate of the function $f(n)$ if there exist $0<a \leq b$ such that $a f(n) \leq \varphi(n) \leq b f(n)$ for all $n \gg 0$.

We can easily achieve growth of $\varphi(n)=\left|S^{R}(\nu) \cap(0, n)\right|$ of the rate $n^{d}$ on a regular local ring $R$ of dimension $d$. Choose $d$ rationally independent real positive numbers $\gamma_{1}, \ldots, \gamma_{d}$, a regular system of parameters $x_{1}, \ldots, x_{d}$ of $R$ and prescribe that $\nu\left(x_{i}\right)=\gamma_{i}$ for all $i$. It is also possible to achieve growth asymptotic to $n^{d}$ from a rational rank 1 valuation, as we show in Example 4.6. We also give examples in this section showing that a wide range of interesting growth can be occured.

We use the following characterization of value semigroups dominating a regular local ring of dimension two of [10], and as may also be found with a different treatment in [5]. We state the characterization in the notation of [3].

Proposition 4.1. Let $S$ be a well ordered subsemigroup of $\mathbb{Q}_{+}$which is not isomorphic to $\mathbb{N}$ and whose minimal system of generators $\left(1, a_{1}, \ldots, a_{i}, \ldots\right)$ is of ordinal type $\leq \omega$. Let $S_{i}$ denote the semigroup generated by $1, a_{1}, \ldots, a_{i}$, and $G_{i}$ the subgroup of $\mathbb{Q}$ which it generates. Let $S_{0}=\mathbb{N}_{+}$and $G_{0}=\mathbb{Z}$. Set $q_{i}=\left[G_{i}: G_{i-1}\right]$ for $i \geq 1$. Let $s_{i}$ be the smallest positive integer $s$ such that $s a_{i} \in S_{i-1}$. Then $S$ is the semigroup $S^{R}(\nu)$ of a valuation $\nu$ dominating a regular local ring $R$ of dimension 2 with algebraically closed residue field if and only if

$$
\text { for each } i \geq 1 \text { we have } s_{i}=q_{i} \text { and } a_{i+1}>q_{i} a_{i} \text {. }
$$

We need the following two statements to estimate the number of terms of a rational rank 1 semigroup $S \subset \mathbb{R}$, which are contained in a fixed interval of length 1 . Our notation is $\mathbb{N}=\mathbb{Z}_{\geq 0}$.

Lemma 4.2. Suppose that $a_{1}, \ldots, a_{k}$ are positive rational numbers. Let $G_{0}=\mathbb{Z}$. For $a$ fixed $1 \leq i \leq k$ let $S_{i}$ be the subsemigroup of $\mathbb{Q}_{\geq 0}$ generated by $1, a_{1}, \ldots, a_{i}$ and $G_{i}$ be the group $S_{i}+\left(-S_{i}\right)$. Suppose that $q_{i}=\left[G_{i}: G_{i-1}\right]$ and $x_{i}=\left(q_{1}-1\right) a_{1}+\cdots+\left(q_{i}-1\right) a_{i}$.

Then for all integers $1 \leq i \leq k$ we have $\left|S_{i} \cap\left(x_{i}-1, x_{i}\right]\right| \geq q_{1} \cdots q_{i}$. Moreover, for all integers $1 \leq i \leq k$ and real numbers $x>x_{i}$ we have $S_{i} \cap[x-1, \infty)=G_{i} \cap[x-1, \infty)$ and $\left|S_{i} \cap[x-1, x)\right|=q_{1} \cdots q_{i}$. 
Proof. Notice that $G_{i}=\frac{1}{q_{1} \cdots q_{i}} \mathbb{Z}$. Thus $S_{i} \cap[x-1, \infty)=G_{i} \cap[x-1, \infty)$ for all $x>x_{i}$ if and only if $\left|S_{i} \cap[x-1, x)\right|=q_{1} \cdots q_{i}$ for all $x>x_{i}$ if and only if $\left|S_{i} \cap[x-1, x)\right| \geq q_{1} \cdots q_{i}$ for all $x>x_{i}$. Also since $S_{i}+\mathbb{N}=S_{i}$, the fact that $\left|S_{i} \cap\left(x_{i}-1, x_{i}\right]\right| \geq q_{1} \cdots q_{i}$ implies $\left|S_{i} \cap(x-1, x]\right| \geq q_{1} \cdots q_{i}$ for all $x \geq x_{i}$. Moreover, if $x$ is a fixed real number, since $S_{i}$ is discrete there exists $\varepsilon_{0}>0$ such that for all $0<\varepsilon \leq \varepsilon_{0}$ the following equality between sets holds $S_{i} \cap[x-1, x)=S_{i} \cap(x-\varepsilon-1, x-\varepsilon]$. Therefore, the fact that $\left|S_{i} \cap\left(x_{i}-1, x_{i}\right]\right| \geq q_{1} \cdots q_{i}$ also implies $\left|S_{i} \cap[x-1, x)\right| \geq q_{1} \cdots q_{i}$ for all $x>x_{i}$.

Assume that $k=1$. Then $S_{1}$ can be presented as a disjoint union of $\mathbb{N}$-modules

$$
S_{1}=\mathbb{N} \cup\left(a_{1}+\mathbb{N}\right) \cup \cdots \cup\left(\left(q_{1}-1\right) a_{1}+\mathbb{N}\right) .
$$

If $0 \leq j \leq\left(q_{1}-1\right)$ then $\left(x_{1}-j a_{1}\right) \geq 0$ and $\left|\left(j a_{1}+\mathbb{N}\right) \cap\left(x_{1}-1, x_{1}\right]\right|=\mid \mathbb{N} \cap\left(x_{1}-j a_{1}-\right.$ $\left.1, x_{1}-j a_{1}\right] \mid=1$. Thus $\left|S_{1} \cap\left(x_{1}-1, x_{1}\right]\right|=q_{1}$.

Assume that $k>1$. By induction it suffices to assume that the statement is true for $i \leq k-1$. Notice that

$$
S_{k} \supset S_{k-1} \cup\left(a_{k}+S_{k-1}\right) \cup \cdots \cup\left(\left(q_{k}-1\right) a_{k}+S_{k-1}\right),
$$

where the union on the right is a disjoint union of $S_{k-1}$-modules.

If $0 \leq j \leq\left(q_{k}-1\right)$ then $\left(x_{k}-j a_{k}\right) \geq x_{k-1}$ and $\left|\left(j a_{k}+S_{k-1}\right) \cap\left(x_{k}-1, x_{k}\right]\right|=$ $\left|S_{k-1} \cap\left(x_{k}-j a_{k}-1, x_{k}-j a_{k}\right]\right| \geq q_{1} \cdots q_{k-1}$. Thus, $\left|S_{k} \cap\left(x_{k}-1, x_{k}\right]\right| \geq q_{1} \cdots q_{k}$.

Corollary 4.3. Under the assumptions of corollary 4.2 suppose also that $a_{i+1}>q_{i} a_{i}$ for all $1 \leq i \leq k-1$. Then $\left|S_{i} \cap[n-1, n)\right|=q_{1} \cdots q_{i}$ for all integers $n \geq q_{i} a_{i}$.

Proof. It suffices to notice that $\left(q_{1}-1\right) a_{1}+\cdots+\left(q_{i}-1\right) a_{i}<q_{i} a_{i}$ in this case.

We will now give examples of value semigroups with unexpected rate of growth of the function $\varphi(n)=\left|S \cap\left(0, n s_{0}\right)\right|$.

Example 4.4. $(n \sqrt{n}$ rate of growth)

Let $R=k[x, y]_{(x, y)}$ where $k$ is an algebraically closed field. Let $\nu$ be a valuation of the quotient field of $R$ defined by its generating sequence $\left\{P_{i}\right\}_{i \geq 0}$ as follows

$$
\begin{array}{ll}
P_{0}=x, & \nu\left(P_{0}\right)=1 \\
P_{1}=y, & \nu\left(P_{1}\right)=4+\frac{1}{2} \\
P_{2}=P_{1}^{2}-x^{9}, & \nu\left(P_{2}\right)=16+\frac{1}{2^{2}} \\
P_{3}=P_{2}^{2}-x^{28} P_{1}, & \nu\left(P_{3}\right)=64+\frac{1}{2^{3}} \\
P_{k+1}=P_{k}^{2}-x^{7 \cdot 4^{k-1}} P_{k-1}, & \nu\left(P_{k+1}\right)=4^{k+1}+\frac{1}{2^{k+1}} .
\end{array}
$$

Denote by $S$ the semigroup $S_{R}(\nu)=\nu\left(m_{R} \backslash\{0\}\right)$. Then $\varphi(n)$ grows like $n \sqrt{n}$.

Proof. We will show that $\frac{1}{6} n \sqrt{n}<\varphi(n)<\frac{4}{3} n \sqrt{n}$.

Set $a_{i}=\nu\left(P_{i}\right)$ for all $i \geq 1$. Then $S$ is a subsemigroup of $\mathbb{Q}_{+}$generated by $1, a_{1}, a_{2}, \ldots$ With notation of Proposition 4.1 we have $s_{i}=q_{i}=2$ for all $i \geq 1$ and $q_{i} a_{i} \leq 2 \cdot 4^{i}+1<$ $4^{i+1}<a_{i+1}$. This shows that $\nu$ is well defined. Also, by corollary 4.3 we find a lower bound on $|S \cap[n-1, n)|$ for $n \geq 4^{i}$ :

$$
|S \cap[n-1, n)| \geq\left|S_{i-1} \cap[n-1, n)\right|=2^{i-1} .
$$

If $n>1$ set $i=\left\lfloor\log _{4} n\right\rfloor$. Then $4^{i} \leq n<4^{i+1}$ and $2^{i-1} \leq|S \cap[n-1, n)| \leq 2^{i}$. Thus for all $n \in \mathbb{N}_{+}$we have

$$
\int_{n-1}^{n} \frac{\sqrt{t}}{4} d t<\frac{\sqrt{n}}{4}<|S \cap[n-1, n)| \leq \sqrt{n}<\int_{n}^{n+1} \sqrt{t} d t .
$$


Then

$$
|S \cap(0, n)|=|S \cap[1, n)|<\int_{2}^{n+1} \sqrt{t} d t=\frac{2}{3}((n+1) \sqrt{n+1}-2 \sqrt{2})<\frac{4}{3} n \sqrt{n}
$$

and

$$
|S \cap(0, n)|=3+|S \cap[4, n)|>3+\int_{4}^{n} \frac{\sqrt{t}}{4} d t>3+\frac{1}{6}(n \sqrt{n}-8)>\frac{1}{6} n \sqrt{n} .
$$

A more precise estimate can be obtained for $n=4^{k}$. By induction on $k \in \mathbb{N}_{+}$we see that $\frac{8^{k}}{3}<\varphi\left(4^{k}\right)<\frac{8^{k}}{2}$, since

$$
\varphi(4)=3, \quad 8 / 3<3<4
$$

and

and

$$
\varphi\left(4^{k+1}\right)=\varphi\left(4^{k}\right)+\left|S \cap\left[4^{k}, 4^{k+1}\right)\right|<\frac{8^{k}}{2}+3 \cdot 2^{k} \cdot 4^{k}<\frac{8^{k+1}}{2}
$$

$$
\varphi\left(4^{k+1}\right)=\varphi\left(4^{k}\right)+\left|S \cap\left[4^{k}, 2 \cdot 4^{k}\right)\right|+\left|S \cap\left[2 \cdot 4^{k}, 4^{k+1}\right)\right|>\frac{8^{k}}{3}+2^{k-1} \cdot 4^{k}+2 \cdot 2^{k} \cdot 4^{k}>\frac{8^{k+1}}{3} .
$$

This example can be generalized to a construction of a value semigroup $S$ such that $\varphi(n)$ grows like a power function $n^{\alpha}$, where $\alpha \in \mathbb{Q}$, with natural restriction $1<\alpha<2$.

Example 4.5. ( $n^{\alpha}$ rate of growth)

Suppose that $0<p<q$ are coprime integers. Let $r=2^{q}$ and $s=2^{p}$. Let $R=k[x, y]_{(x, y)}$ where $k$ is an algebraically closed field. Let $\nu$ be a valuation of the quotient field of $R$ defined by its generating sequence $\left\{P_{i}\right\}_{i \geq 0}$ as follows

$$
\begin{array}{ll}
P_{0}=x, & \nu\left(P_{0}\right)=1 \\
P_{1}=y, & \nu\left(P_{1}\right)=r+\frac{1}{s} \\
P_{2}=P_{1}^{s}-x^{s r+1}, & \nu\left(P_{2}\right)=r^{2}+\frac{1}{s^{2}} \\
P_{3}=P_{2}^{s}-x^{(s r-1) r} P_{1}, & \nu\left(P_{3}\right)=r^{3}+\frac{1}{s^{3}} \\
P_{k+1}=P_{k}^{s}-x^{(s r-1) r^{k-1}} P_{k-1}, & \nu\left(P_{k+1}\right)=r^{k+1}+\frac{1}{s^{k+1}} .
\end{array}
$$

Denote by $S$ the semigroup $S_{R}(\nu)=\nu\left(m_{R} \backslash\{0\}\right)$. Then $\varphi(n)$ grows like $n^{1+p / q}$.

Proof. Set $a_{i}=\nu\left(P_{i}\right)$ for all $i \geq 1$. Then $S$ is a subsemigroup of $\mathbb{Q}_{+}$generated by $1, a_{1}, a_{2}, \ldots$. With notation of Proposition 4.1 we have $s_{i}=q_{i}=s$ for all $i \geq 1$ and $q_{i} a_{i} \leq s \cdot r^{i}+1<r^{i+1}<a_{i+1}$. This implies that $\nu$ is well defined. Also, by corollary 4.3 we find a lower bound on $|S \cap[n-1, n)|$ for $n \geq r^{i}$ :

$$
|S \cap[n-1, n)| \geq\left|S_{i-1} \cap[n-1, n)\right|=s^{i-1} .
$$

If $n>1$ set $i=\left\lfloor\log _{r} n\right\rfloor$. Then $r^{i} \leq n<r^{i+1}$ and $s^{i-1} \leq|S \cap[n-1, n)| \leq s^{i}$. Thus since $s^{i}=\left(r^{i}\right)^{p / q}$ and $s^{i-1}=\frac{\left(r^{i+1}\right)^{p / q}}{s^{2}}$ for all $n \in \mathbb{N}_{+}$we have

$$
\int_{n-1}^{n} \frac{t^{p / q}}{s^{2}} d t<\frac{n^{p / q}}{s^{2}}<|S \cap[n-1, n)| \leq n^{p / q}<\int_{n}^{n+1} t^{p / q} d t .
$$

Then

$$
|S \cap(0, n)|=|S \cap[1, n)|<\int_{2}^{n+1} t^{p / q} d t=\frac{q}{p+q}\left((n+1)^{1+p / q}-2^{1+p / q}\right)<\frac{3 q}{p+q} n^{1+p / q}<3 n^{1+p / q}
$$


and

$$
|S \cap(0, n)|=r-1+\mid S \cap[r, n)) \mid>r-1+\int_{r}^{n} \frac{t^{p / q}}{s^{2}} d t=r-1+\frac{q}{s^{2}(p+q)}\left(n^{1+p / q}-r s\right)>\frac{n^{1+p / q}}{2 s^{2}} .
$$

That is $\frac{1}{2 s^{2}} n^{1+p / q}<\varphi(n)<3 n^{1+p / q}$.

We remark that in the above construction it is necessary to have the strict inequality $p<q$. However, the maximal rate of growth of $n^{2}$ is also achievable on a rational rank 1 semigroup of a valuation centered in a 2-dimensional polynomial ring, as we show in the next example.

Example 4.6. ( $n^{2}$ rate of growth)

Let $R=k[x, y]_{(x, y)}$ where $k$ is an algebraically closed field. Let $\nu$ be a valuation of the quotient field of $R$ defined by its generating sequence $\left\{P_{i}\right\}_{i \geq 0}$ as follows

$$
\begin{array}{lrl}
P_{0}=x, & \nu\left(P_{0}\right)=1 \\
P_{1}=y, & \nu\left(P_{1}\right)=1+\frac{1}{2} \\
P_{2}=P_{1}^{2}-x^{2+1}, & \nu\left(P_{2}\right)=2+1+\frac{1}{2^{2}} \\
P_{3}=P_{2}^{2}-x^{2^{2}+2-1} P_{1}, & \nu\left(P_{3}\right)=2^{2}+2+\frac{1}{2}+\frac{1}{2^{3}} \\
P_{k+1}=P_{k}^{2}-x^{2^{k}+2^{k-1}-2^{k-2}} P_{k-1}, & \nu\left(P_{k+1}\right)=2^{k}+2^{k-1}+2^{k-3}+\cdots+2^{-k-1} . \\
\text { Denote by } S \text { the semigroup } S_{R}(\nu)=\nu\left(m_{R} \backslash\{0\}\right) . \text { Then } \varphi(n) \text { grows like } n^{2} .
\end{array}
$$

Proof. Set $a_{i}=\nu\left(P_{i}\right)$ for all $i \geq 1$. Then $S$ is the subsemigroup of $\mathbb{Q}_{+}$generated by $1, a_{1}, a_{2}, \ldots$ We have $q_{i}=2$ for all $i \geq 1$. Solving the recursion relation, we have

$$
a_{i}=2^{i-1}+\frac{1}{3}\left(2^{i}-\frac{1}{2^{i}}\right)
$$

for $i \geq 1$. We have $q_{i-1} a_{i-1}=2 a_{i-1}=\frac{5}{6} 2^{i}-\frac{1}{3} \frac{1}{2^{i-2}}<2^{i}$. Corollary 4.3 shows that

$$
|S \cap[n-1, n)| \geq\left|S_{i-1} \cap[n-1, n)\right|=2^{i-1}
$$

for $n \geq 2^{i}$. For $n>1$, set $i=\left\lfloor\log _{2} n\right\rfloor$, so that $2^{i} \leq n<2^{i+1}$. We have

$$
\frac{1}{4} \int_{n-1}^{n} t d t<\frac{n}{4}<\frac{2^{i+1}}{4}=2^{i-1} \leq|S \cap[n-1, n)| \text {. }
$$

Thus for $n \geq 4$,

$$
|S \cap(0, n)|=3+|S \cap[4, n)|>3+\frac{1}{4} \int_{4}^{n} t d t>\frac{n^{2}}{8} .
$$

Since $|S \cap(0, n)|<\left(\begin{array}{c}1+n \\ 2\end{array}\right)$ by Corollary 2.3, $\varphi(n)$ grows at the rate of $n^{2}$.

Another interesting example is of logarithmic growth.

Example 4.7. ( $n \log _{10} n$ rate of growth)

Let $R=k[x, y]_{(x, y)}$ where $k$ is an algebraically closed field. Let $\nu$ be a valuation of the quotient field of $R$ defined by its generating sequence $\left\{P_{i}\right\}_{i \geq 0}$ as follows

$$
\begin{aligned}
& P_{0}=x, \quad \nu\left(P_{0}\right)=1 \\
& P_{1}=y, \quad \nu\left(P_{1}\right)=10+\frac{1}{2} \\
& P_{2}=P_{1}^{2}-x^{21}, \quad \nu\left(P_{2}\right)=10^{2}+\frac{1}{2^{2}} \\
& P_{3}=P_{2}^{2}-x^{190} P_{1}, \quad \nu\left(P_{3}\right)=10^{4}+\frac{1}{2^{3}} \\
& P_{k+1}=P_{k}^{2}-x^{\alpha(k)} P_{k-1}, \quad \nu\left(P_{k+1}\right)=10^{2^{k^{3}}}+\frac{1}{2^{k+1}}, \quad \alpha(k)=2 \cdot 10^{2^{k-1}}-10^{2^{k-2}} .
\end{aligned}
$$

Denote by $S$ the semigroup $S_{R}(\nu)=\nu\left(m_{R} \backslash\{0\}\right)$. Then $\varphi(n)$ grows like $n \log _{10} n$. 
Proof. If $n \geq 10$ let $k=\left\lfloor\log _{2} \log _{10} n\right\rfloor$. Then $10^{2^{k}} \leq n<10^{2^{k+1}}$ and $2^{k} \leq|S \cap[n-1, n)| \leq$ $2^{k+1}$. Thus for all $n \geq 10$ we have

$$
\int_{n-1}^{n} \frac{\log _{10} t}{2} d t<\frac{\log _{10} n}{2} \leq|S \cap[n-1, n)| \leq 2 \log _{10} n<\int_{n}^{n+1} 2 \log _{10} t d t
$$

and

$$
\frac{n \log _{10} n}{4}<9+\int_{10}^{n} \frac{\log _{10} t}{2} d t<|S \cap[0, n)|<9+\int_{11}^{n+1} 2 \log _{10} t d t<2 n \log _{10} n .
$$

That is $\frac{1}{4} n \log _{10} n<\varphi(n)<2 n \log _{10} n$.

\section{When is A SEMigroup A VAlUe SEMigroup?}

Corollary 2.2 gives a necessary condition for a rank 1 well ordered semigroup $S$ consisting of positive elements of $\mathbb{R}$ to be the value semigroup $S^{R}(\nu)$ of a valuation dominating some local domain $R$. The condition is:

$$
\text { There exists } c>0 \text { and } d \in \mathbb{Z}_{+} \text {such that }\left|S \cap\left(0, n s_{0}\right)\right|<c n^{d} \text { for all } n
$$

where $s_{0}$ is the smallest element of $S$. An interesting question is if (6) is in fact sufficient. (6) is sufficient in the case when $d=1$, as we now show. Suppose that $S \subset \mathbb{R}_{+}$is a semigroup consisting of positive elements which contains a smallest element $s_{0}$. Suppose that there exists $c>0$ such that

$$
\mid S \cap\left[\left(0, n s_{0}\right) \mid<c n\right.
$$

for all $n \in \mathbb{N}$. By Lemma 5.2 below, we may assume that $S \subset \mathbb{Q}_{+}$is finitely generated by some elements $\lambda_{1}, \ldots, \lambda_{r}$. There exists $\alpha \in \mathbb{Q}_{+}$such that there exists $a_{i} \in \mathbb{N}_{+}$with $\lambda_{i}=\alpha a_{i}$ for $1 \leq i \leq r$, and $\operatorname{gcd}\left(a_{1}, \ldots, a_{r}\right)=1$. Let $k[t]$ be a polynomial ring over a field $k$. Let $\nu(f(t))=\alpha \operatorname{ord}(f(t))$ for $f(t) \in k[t]$. $\nu$ is a valuation of $k(t)$. Let $R$ be the one dimensional local domain

$$
R=k\left[t^{a_{1}}, \ldots, t^{a_{r}}\right]_{\left(t^{a_{1}}, \ldots, t^{a_{r}}\right)} .
$$

The quotient field of $R$ is $k(t)$, and $\nu$ dominates $R$. We have that $S=\nu\left(m_{R} \backslash\{0\}\right)=S_{R}(\nu)$.

Lemma 5.1. Suppose that $S \subset \mathbb{R}_{+}$is a semigroup consisting of positive elements which contains a smallest element $s_{0}$. Suppose that there exists $c>0$ and $d \in \mathbb{N}_{+}$such that

$$
\left|S \cap\left(0, n s_{0}\right)\right|<c n^{d}
$$

for all $n \in \mathbb{N}$. Then $S$ is well ordered of ordinal type $\omega$ and has rational rank $\leq d$.

Proof. The fact that $S$ is well ordered of ordinal type $\omega$ is immediate from (8) .

We will prove that the rational rank of $S$ is $\leq d$. After rescaling $S$ by multiplying by $\frac{1}{s_{0}}$, we may assume that $s_{0}=1$. Suppose that $t \in \mathbb{N}$ and $S$ has rational rank $\geq t$. Then there exist $\gamma_{1}, \ldots, \gamma_{t} \in S$ which are rationally independent. Let $b \in \mathbb{N}$ be such that $\max \left\{\gamma_{1}, \ldots, \gamma_{t}\right\}<b$. For $e \in \mathbb{R}_{+}$, we have

$$
\begin{aligned}
|S \cap(0, e)| & \geq \mid\left\{a_{1} \gamma_{1}+\cdots+a_{t} \gamma_{t} \mid a_{1}, \ldots, a_{t} \in \mathbb{N} \text { and } a_{1} \gamma_{1}+\cdots+a_{t} \gamma_{t}<e\right\} \mid-1 \\
& \geq \mid\left\{a_{1} \gamma_{1}+\cdots+a_{t} \gamma_{t} \mid a_{i} \in \mathbb{N} \text { and } 0 \leq a_{i}<\frac{e}{t b} \text { for } 1 \leq i \leq t\right\} \mid-1 \\
& =\mid\left\{\left(a_{1}, \ldots, a_{t}\right) \in \mathbb{N}^{t} \mid 0 \leq a_{i}<\frac{e}{t b} \text { for } 1 \leq i<t\right\} \mid-1
\end{aligned}
$$

since $\gamma_{1}, \ldots, \gamma_{t}$ are rationally independent.

For $a \in \mathbb{N}$ let $n=a b t$. Then we see that

$$
|S \cap(0,(n+1))| \geq a^{t}-1=\left(\frac{1}{b t}\right)^{t} n^{t}-1 .
$$


By (8), we see that $t \leq d$.

Lemma 5.2. Suppose that $S \subset \mathbb{R}_{+}$is a semigroup consisting of positive elements which contains a smallest element $s_{0}$. Suppose that there exists $c>0$ such that

$$
\left|S \cap\left(0, n s_{0}\right)\right|<c n
$$

for all $n \in \mathbb{N}$. Then $S$ is finitely generated, and the group generated by $S$ is isomorphic to $\mathbb{Z}$.

Proof. By Lemma 5.1, $S$ has rational rank 1, so we may assume that $S$ is contained in $\mathbb{Q}_{+}$. We may further assume that $s_{0}=1$. Suppose that $S$ is not finitely generated. Then for $e \in \mathbb{N}$, we can find $\lambda_{1}, \ldots, \lambda_{e} \in S$ such that $\lambda_{i}=\frac{a_{i}}{b_{i}}$ with $a_{i}, b_{i} \in \mathbb{N}_{+}, b_{i}>1$ for all $i$, $\operatorname{gcd}\left(a_{i}, b_{i}\right)=1$ for all $i$ and $b_{1}, \ldots, b_{e}$ all distinct.

There exist $m_{i}, n_{i} \in \mathbb{Z}$ such that $m_{i} a_{i}+n_{i} b_{i}=1$ for $1 \leq i \leq e$. Either $m_{i}>0$ and $n_{i}<0$, or $m_{i}<0$ and $n_{i}>0$. If $m_{i}>0$, then $\left|m_{i}\right| \lambda_{i}=\left|n_{i}\right|+\frac{1}{b_{i}}$. If $m_{i}<0$, then $\left|m_{i}\right| \lambda_{i}=\left|n_{i}\right|-\frac{1}{b_{i}}$. Let $n_{0}=\max \left\{\left|n_{i}\right|+1 \mid 1 \leq i \leq i\right\}$. For $n \geq n_{0}$, we have that $n+\frac{1}{b_{i}} \in S \cap(n, n+1)$ if $m_{i}>0$, and $(n+1)-\frac{1}{b_{i}} \in S \cap(n, n+1)$ if $m_{i}<0$. Thus $|S \cap(n, n+1)| \geq e$ for $n \geq n_{0}$, which implies that

$$
|S \cap(n, n+1)| \geq e n-e n_{0}
$$

for $n \geq n_{0}$. For $e>c$, we have a contradiction to (9).

\section{REFERENCES}

[1] S. Abhyankar, On the valuations centered in a local domain, Amer. J. Math. 78 (1956), 321 - 348.

[2] W. Bruns and J. Herzog, Cohen-Macaulay Rings, revised edition, Cambridge University Press, Cambridge 1996.

[3] S.D. Cutkosky and B. Teissier, Semigroups of valuations on local rings, to appear in Mich. Math. J, arXiv:0801.0449

[4] S.D. Cutkosky and B. Teissier, Semigroups of valuations on local rings, II, arXiv:0805.3788

[5] C. Favre and M. Jonsson, The valuative tree, Lecture Notes in Math 1853, Springer Verlag, Berlin, Heidelberg, New York, 2004.

[6] W. Heinzer and J. Sally, Extensions of valuations to the completion of a local domain, Journal of Pure and Applied Algebra 71 (1991), 175 - 185.

[7] F.-V. Kuhlmann, Value groups, residue fields, and bad places of algebraic function fields, Trans. Amer. Math. Soc. 40 (1936), 363 - 395.

[8] S. MacLane, A construction for absolute values in polynomial rings, Trans. Amer. Math. Soc. 40 (1936), $363-395$.

[9] S. MacLane and O. Schilling, Zero-dimensional branches of rank 1 on algebraic varieties, Annals of Math. 40 (1939), 507 - 520.

[10] M. Spivakovsky, Valuations in function fields of surfaces, Amer. J. Math. 112 (1990), 107 - 156.

[11] B. Teissier, Valuations, deformations, and toric geometry, Proceedings of the Saskatoon Conference and Workshop on valuation theory (second volume), F.-V. Kuhlmann, S. Kuhlmann, M. Marshall, editors, Fields Institute Communications, 33, 2003, 361 - 459.

[12] O. Zariski and P. Samuel, Commutative Algebra Volume II, D. Van Nostrnad, Princeton, 1960.

Steven Dale Cutkosky, Department of Mathematics, 328 Mathematical Sciences Bldg, University of Missouri, Columbia, MO 65211 USA

E-mail address: cutkoskys@missouri.edu

Kia Dalili, Department of Mathematics, 103 Mathematical Sciences Bldg, University of Missouri, Columbia, MO 65211 USA

E-mail address: kia@math.missouri.edu 
Olga Kashcheyeva, Department of Mathematics, University of Illinois, Chicago, Chicago, IL 60607 USA

E-mail address: olga@math.uic.edu 